\title{
Name Index
}

Adorno, Theodor W. 62

Aira, César 193f., 203-215

Anna Amalia (Duchess of Weimar) 260

Antrechaus, Jean d' 284-286

Aragon, Louis 23

Argillet, Stéphane 91

Bachelard, Gaston 300

Baggesen, Jens 236, 239

Bahr, Hans-Dieter 131

Bakhtin, Mikhail 184, 257

Balke, Friedrich 327

Barthes, Roland 229 f., 235, 243

Baudrillard, Jean 131

Bayle, Pierre 228

Becher, Alexander 63-66

Benedetti, Alessandro 283

Benedict, Barbara 244, 250

Benjamin, Walter 30n, 297, 300f., 311, 314, 324

Berend, Eduard 239

Bergson, Henri 319

Besançon, Georges 111f., 114-116

Bjerknes, Vilhelm 122

Blanqui, Auguste 25, 32, $37 \mathrm{f}$.

Boehm, Gottfried 265

Borges, Jorge Luis 226

Bourdieu, Pierre 319, 327

Bouterwek, Friedrich Ludewig 225

Bowker, Geoffrey 77n

Brecht, Bertolt 61, 63, 70-72

Bühler, Karl 196

Buonarroti, Philippe 37

Bureau, Robert $117 f$.

Calfucurá 53

Callou, G. 32

Campe, Rüdiger 227, 237n

Canetti, Elias 30

Canguilhem, Georges 300

Canler, Louis 29

Cassirer, Ernst 196

Caussidière, Marc 23f., 45

Celan, Paul 62
Cerebotini, Luigi 117

Certeau, Michel de 211

Cervantes, Miguel de 206

Charles X 29

Chun, Wendy 318-322, 327

Cooper, Charlotte 185

Corday, Charlotte 247f., 260

Corngold, Stanley 307

Cunliffe, Barry 150, 156n, 161

d'Alembert, Jean-Baptiste le Rond 229n, 269

D'Hondt, Jacques $283 f$.

Danius, Sara 243

Darwin, Charles 207n

Daumier, Honoré 36

Davies, John 139

Davis, Mike 100-102

De Landa, Manuel $11 \mathrm{f}$.

Debord, Guy 42

Defoe, Daniel $1 \mathrm{f} .$, 5, 8, 245

Déjacque, Joseph 31f., 40

Deleuze, Gilles 12, 53, 290, 308n, 319

Dercum, Francis X. 186

Derrida, Jacques 131, 220n, 308, 310

Diderot, Denis 225n, 228f., 269, 281f., 289f.

Digard, Jean-Pierre 145, $155 \mathrm{f}$.

Dreyfus, Alfred 307

Du Camp, Maxime 41

Dumas, George 194

Durkheim, Émile 194, 197n

Edward III 153, 158

Elizabeth I 139

Engels, Friedrich 27, 32, 37

Ersch, Johann Samuel 229

Fitzpatrick, Matthew P. 146

Flatley, Jonathan $223 f$.

Flaubert, Gustave $41,198 \mathrm{n}$

Fonvielle, Wilfrid 115

Foucault, Michel 13n, 33, 35, 92, 136, 225 , 238, 257, 290, 325

Fourier, Charles 31, 40

Fracastoro, Girolamo 238 
Frankopan, Peter 150-153

Freud, Sigmund 163

Gaillard, Napoléon (“Gaillard père”) 39-42

Gamman, Lorraine 104

Garve, Christian 287

Gazagnadou, Didier 158, 162

Gellert, Christian Fürchtegott 223

Gibbon, Edward 289

Gilman, Sander L. 177, 307, 313n

Girard, René 138, 279

Glaisher, James $111 f$.

Goethe, Johann Wolfgang von 198, 260, 281, 290

Goody, Jack 150

Gracq, Julien 154

Grillon, Edme Jean Louis 32

Grimm, Jakob und Wilhelm 78

Gruber, Johann Gottfried 229

Guattari, Félix 12, 53, 308n

Hamilton, John 311

Hartmann, Karl 84f.

Haudricourt, André-Georges 141, 156n

Haussmann, Georges-Eugène 25, 33, 35, 37, 42, 44

Hebel, Johann Peter 247

Hederich, Benjamin 228

Hegel, Georg Wilhelm Friedrich 224, 235f., 277-294

Heidegger, Martin 135

Heinse, Gottlob Heinrich 229

Hennion, Antoine 75n, 139

Hergesell, Hugo 118n, 122

Hermite, Gustave 111-116, 120n

Herodotus $54 \mathrm{f}$.

Hilliard, Nicholas 140

Holbach, Paul Thiry d' 280

Honan, Mat 327f.

Humboldt, Alexander von 194, 204

Hutchin, Edwin 76

Ingold, Timothy 135

Jacobi, Friedrich Heinrich 247

Jacobs, Jane 100

Jacoubet, Théodore 32
James, William 319

Janet, Pierre 194

Jean Paul 219-241, 243-261

Jeanne, Charles 29

Joyce, James 243

Kafka, Franz 191, 295-315

Kant, Immanuel 225, 231n, 300n

Kapp, Ernst 163

Kircher, Athanasius 283

Kirkman, Marshall M. 83

Kittler, Friedrich 3f., 131-133, 135, 219

Kittler, Wolf 308

Kleist, Heinrich von $118-120$

Kluge, Alexander 131

Knigge, Adolph Freiherr von 284

König, René 197

Krünitz, Johann Georg 228

Lacan, Jacques 137, 292

Latour, Bruno 12, 14, 49, 94-96, 136, 156n, 173n, 249f., 300n

Lecouturier, Henri 32

Leroi-Gourhan, André 6n, 10n, 75n, 145, 156n, 159

Lévi-Strauss, Claude $196 f$.

Lichtenberg, Georg Christoph 221n

Livy $136 f$.

Luxemburg, Rosa 292

Macho, Thomas 295

Marat, Jean Paul 261

Marco Polo 150

Marville, Charles 36

Marx, Karl 37

Maurer, Julius $121 \mathrm{f}$.

Mauss, Marcel 2f., 6n, 9f., 13, 75n, 131, 156n, 159f., 187-215

Maye, Harun 189, 194, $212 \mathrm{f}$.

McLuhan, Marshall 163

Meckel, Johann Friedrich 257

Merck, Klemens 174

Merruau, Charles 35

Mitre, Bartolomé 53

Monroe, Marilyn 324

Montaigne, Michel de 226f., 231

More, Thomas 53 
Morgan, Thomas Hunt 299

Muybridge, Eadweard 177-186

Nadar, Félix 116

Nadaud, Martin 30f., 36

Nancy, Jean-Luc 322

Napoleon III 32, 37

Negt, Oskar 131

Neumann, Peter Horst 254

Neumayer, Georg von 68

Newman, Oscar 100

Newton, Isaac 259

Nietzsche, Friedrich 289

Norman, Nils $103 \mathrm{f}$.

Paré, Ambroise 279

Pariser, Eli 320f., 328n, 329

Paté, Gilles 91

Paviot, Jacques 153

Pethes, Nicolas 187n, 199, 203, 212

Pliny (the Elder) 146f., 223n

Plutarch 136f.

Pope, Alexander 244, 258n

Price, Leah 249

Rabelais, François 184-186

Ravaisson, Felix 319

Reimarus, Johann Albert Heinrich $285 f$.

Rheinberger, Hans-Jörg 298-300

Richardson, Lewis Fry 122-125

Richter, Johann Paul Friedrich (see Jean Paul)

Robida, Albert 42

Rosas, Juan Manuel de 51

Ross, Sir John 64

Rousseau, Jean-Jacques 5

Rouvroy, Antoinette 325

Rugendas, Georg Philip 207

Rugendas, Moritz 194, 204-215

Rustichello da Pisa 150

Ryan, Simon 307

Sarmiento, Domingo Faustino 47-59

Schäfer, Armin 238, 254, 257

Schivelbusch, Wolfgang 131

Schlegel. Friedrich $225 \mathrm{n}$
Schlegel, Gustaaf 152-154

Schmidt-Hannisa, Hans-Walter 234

Schmitt, Carl 50, $52 \mathrm{f}$.

Scholl, Ewald Friedrich 82

Schott, Gerhard 68

Schuiten, François 124

Schupbach, William 185

Schüttpelz, Erhard 75n, 187f., 190, 192, 199

Seckendorff, Leopold von 206

Seneca 146f., 154

Serres, Michel 136-138

Shaftesbury (Anthony Ashley Cooper, Earl of Shaftesbury) 244

Siegert, Bernhard 79n, 172

Silbermann, Albert 117

Sklovskij, Victor 296

Star, Susan Leigh $75 f$.

Starr, S. Frederick 150

Sterne, Laurence 245

Stockhammer, Robert 232n, 265

Swenson, Gene 323

Swift, Jonathan 245

Tarde, Gabriel 290, 319, 326f.

Teisserenc de Bort, Léon-Philippe 116

Vanhöffen, Ernst 170, 172, 174

Vespucci, Amerigo 54

Viollet-le Duc, Eugène 153

Vismann, Cornelia 6n, 11, 23, 309n

Voigt, Johann Gustav $250 f$.

Warhol, Andy $323 \mathrm{f}$.

Wheatstone, Charles 116

White, Lynn $157 f$.

Whyte, William H. 99-102, 105

Widlok, Thomas 76

Winthrop-Young, Geoffrey 134, 187

Yersin, Alexandre 283

Zedelmaier, Helmut $264 \mathrm{n}$

Zischler, Hans 243

Žižek, Slavoj 282, 287, 292

Zola, Émile 36 
